\title{
1 Analysis of the multi-copy gene family FAM90A as a copy number variant in different ethnic backgrounds
}

\author{
Nina Bosch ${ }^{\mathrm{a}, \mathrm{b}}$, Geòrgia Escaramís ${ }^{\mathrm{a}, \mathrm{b}}$, Josep M. Mercader ${ }^{\mathrm{a}, \mathrm{b}}$, Lluís Armengol ${ }^{\mathrm{a}, \mathrm{b}}$, Xavier Estivill ${ }^{\mathrm{a}, \mathrm{b}, \mathrm{c}, *}$ \\ a Genetic Causes of Disease Group, Genes and Disease Programme Center for Genomic Regulation (CRG-UPF), Barcelona, Catalonia, Spain \\ ${ }^{\mathrm{b}}$ CIBER en Epidemiología y Salud Pública (CIBERESP), Barcelona, Catalonia, Spain \\ c Department of Health and Experimental Life Sciences, Pompeu Fabra University (UPF), Barcelona, Catalonia, Spain
}

\section{A R T I C L E I N F O}

\section{Article history:}

Received 18 March 2008

Received in revised form 30 April 2008

Accepted 2 May 2008

Available online $\mathrm{xxxx}$

Received by M. D'Urso

\section{Keywords:}

FAM90A (family with sequence similarity 90

GenBank: NM_018088)

Inter-population variability

Real-time PCR

\begin{abstract}
A B S T R A C T
Copy number variants contribute extensively to inter-individual genomic differences, but little is known about their inter-population variability and diversity. In a previous study (Bosch et al., 2007; 16:2572-2582), we reported that the primate-specific gene family FAM90A, which accounts for as many as 25 members in the human reference assembly, has expanded the number of FAM90A clusters across the hominoid lineage. Here we examined the copy number variability of FAM90A genes in 260 HapMap samples of European, African, and Asian ancestry, and showed significant inter-population differences $(p<0.0001)$. Based on the recent study of Stranger et al. (2007; 315:848-853), we also explored the correlation between copy number variability and expression levels of the FAM90A gene family. Despite the high genomic variability, we found a low correlation between FAM90A copy number and expression levels, which could be due to the action of independent trans-acting factors. Our results show that FAM90A is highly variable in copy number between individuals and between populations. However, this variability has little impact on gene expression levels, thus highlighting the importance of genomic variability for genes located in regions containing segmental duplications.
\end{abstract}

(C) 2008 Elsevier B.V. All rights reserved.

\section{Introduction}

Nucleotide and gene copy diversity is an important driving force of organism evolution and also of selection within any given species (Nguyen et al., 2006; Norton et al., 2007). Genetic variation occurs at multiple levels, from single nucleotide polymorphisms (SNPs), to structural changes such as inversions, deletions or duplications of large DNA fragments, commonly known as copy number variants (CNVs). Recently, it has been shown that BAC array studies have led to an over-estimation of the size of CNVs, and therefore fine-scale analysis of the architecture of these CNVs is necessary (Perry et al., 2008). Although many efforts are needed to elucidate the impact of CNVs on gene expression, recent work has indicated a contribution of both SNPs and CNVs to the variability in the expression of human genes (Stranger et al., 2007).

Abbreviations: SNP, single nucleotide polymorphism; CNV, copy number variant $\mathrm{SD}$, segmental duplication; REPD, distal segmental duplications on 8p23.1; REPP, proximal segmental duplications on 8p23.1; UPL, universal probe library; $\mathrm{Cp}$, crossing point.

* Corresponding author. Genes and Disease Programme, Centre for Genomic Regulation (CRG), Plaça Charles Darwin s/n, (Carrer Dr Aiguader, 88), Room 521, PRBB Building, 08003 Barcelona, Catalonia, Spain. Tel.: +3493 316 0159; fax: +3493 3160099.

E-mail address: xavier.estivill@crg.es (X. Estivill).
Genomic copy number gains and losses could have a major impact 43 on gene expression by directly modifying mRNA levels, interrupting a 44 gene transcript, or by having positional effects on regulatory regions 45 (Beckmann et al., 2007; Estivill and Armengol, 2007). Several copy 46 number changes have been found to be related to disease phenotypes, 47 such as the low copy number of FCGR3B associated with a predis- 48 position to develop glomerulonephritis (Aitman, 2006) and auto- 49 immune disorders (Fanciulli et al., 2007), whilst a high copy number of 50 CCL3L1 could have a protective role against HIV infection (Gonzalez 51 et al., 2005) (reviewed in Estivill and Armengol, 2007). Some of these 52 CNVs have been analyzed in different populations and inter-popula- 53 tion analyses have revealed a wide variability in copy number 54 (Gonzalez et al., 2005; Perry et al., 2007; Jakobsson et al., 2008). 55 Thus, CCL3L1 and AMY1 show striking differences depending on the 56 population examined, indicating that variability in copy number has 57 probably contributed to adaptation to different environments (Gon- 58 zalez et al., 2005; Perry et al., 2007). Therefore, it is important to 59 characterize the variability of CNVs amongst different populations and 60 to evaluate their consequences at the expression level.

The generation of multi-copy gene families is an important factor 62 in the generation of biological novelty (Lynch and Conery, 2000). 63 Multi-copy gene families could be generated as a result of unequal 64 crossover processes between segmental duplications (SDs). Several 65 multi-copy gene families, such as olfactory receptor genes or defensin 66 clusters, are located along the distal SDs on chromosome 8p23.1. Copy 67 
number variability in the case of defensins has been well established. For alpha defensins DEFA1/3 variation can range from 4 to 14 tandem array repeats, with $10 \%$ of the individuals completely lacking DEFA3 (Aldred et al., 2005; Linzmeier and Ganz, 2005; Ballana et al., 2007). The beta defensin gene cluster ranges from 2 to 12 copies per diploid genome (Hollox et al., 2003). Recent studies have revealed that individuals with three or less copies of the DEFB4 gene have a higher risk to develop Crohn's disease (Fellermann et al., 2006). Similarly, individuals with higher genomic copy number of beta defensin genes show association with psoriasis predisposition (Hollox et al., 2008).

Here we analyze the copy number variability of FAM90A, a primate-specific gene family, which accounts for as many as 25 members per haploid genome. This family has undergone expansion during primate evolution, and it is located in an extremely dynamic region of the human genome regarding structural variation. We have studied inter and intra-population variability in 260 subjects from three different ethnic backgrounds and have explored the correlation between FAM90A copy number and its expression levels.

\section{Materials and methods}

\subsection{Samples analyzed}

Samples included in the study were 87 residents from Utah with ancestry from northern and western Europe (CEU); 87 individuals, Han Chinese from Beijing (CHB) and Japanese from Tokyo (JPT); and 86 Yoruba individuals from Ibadan (YRI), Nigeria.

\subsection{Sequence similarity searches}

To establish the nucleotide identity level between the 25 members of the FAMOA gene family, we performed BLAT and BLAST searches using the FAM90A1 member on chromosome 12p13 as a query. The same method was used to map the four different clusters of FAM90A genes located on the 8p23.1 distal duplications (REPD), and the single copies on the proximal duplicons (REPP).

\subsection{Real-time PCR analysis}

For the quantitative real-time PCR amplification, three sets of universal probe library (UPL) probes and primer pairs were used, one probe targeting the second exon of FAM90A genes (UPL probe \#79), another probe targeting exon 6 (UPL probe \#29) and another targeting the RNAseP, a single copy gene (UPL probe \#14) used to normalize FAM90A values. Probes and primer sets were designed at the ProbeFinder Design assay center website (https://www.rocheapplied-science.com/sis/rtpcr/upl/adc.jsp). Real-time PCR was performed in the LightCycler ${ }^{\circledR} 480$ System (Roche Molecular Diagnostics), using the following program conditions for both amplicons: $10 \mathrm{~min}$ of pre-incubation at $95^{\circ} \mathrm{C}$ followed by 45 cycles of $15 \mathrm{~s}$ at $95^{\circ} \mathrm{C}, 1 \mathrm{~min}$ at $59{ }^{\circ} \mathrm{C}$ and $30 \mathrm{~s}$ at $72{ }^{\circ} \mathrm{C}$. For each $10 \mu \mathrm{l}$ sample in a 384 -multiwell plate (Roche Molecular Diagnostics), individual reactions were carried out in triplicate according to the manufacturer's instructions. Independent genomic DNA-based standard curves were used to determine the efficiencies of FAM90A target amplifications. Estimates of FAM90A copies quantification were obtained in the form of crossing point (Cp) values based on the 'second derivative maximum' method as computed by the LightCycler® 480 (Roche Molecular Diagnostics). Further data analysis was performed with the $\mathrm{Cp}$ raw data as described by Pfaffl (2001).

\subsection{Statistical analysis}

Normalized gene expression values from the three different populations (CEU, CHB/JPT and YRI) were downloaded from the Sanger Genevar webpage (http://www.sanger.ac.uk/humgen/genevar/). Data retrieved by the Illumina ${ }^{\mathrm{TM}}$ probe GI_42658889-S, which hybridizes to 125 FAM90A members, was used to explore the correlation between 126 FAM90A expression levels and FAM90A copy number. 127

Individuals included in the analysis were the same as for the 128 FAM90A copy number study. Since the $\log _{2}$ values corresponding to 129 the two real-time quantitative assays were highly equivalent, we used 130 the average values of the two probes to explore association between 131 gene dosage and expression levels. To determine inter-population 132 differences in relation to FAM90A copy number, a linear model was 133 used, including the gender as a confounding variable and a correlation 134 structure among individuals from the same trio in an attempt to 135 capture dependence, if any, between parents and their offspring. The 136 correlation model structure is included in the model by specifying the 137 appropriate total variance-covariance pattern matrix. No statistically 138 significant differences between males and females were found. The 139 same approach was used to detect association between expression 140 levels and FAM90A copy number. Here we included gender, origin and 141 the interaction between both variables with FAM90A copy numbers to 142 see whether the relationship between expression and FAM90A copy 143 numbers differed according to inter-population variables and/or 144 gender differences. A correlation structure was also specified to 145 capture dependence among individuals from the same family. None of 146 the interactions were statistically significant as well as gender and 147 origin effects by themselves; therefore results are shown as a simple 148 linear association between expression levels and FAM90A copy 149 numbers. All the analysis was performed using the MIXED procedure 150 from SAS System vs. 9.0.

3.1. Sequence similarity and genomic distribution of FAM90A gene family 153

We have recently characterized a novel multi-copy gene family, 154 FAM90A, widely expressed in the 14 tissues we tested, which included 155 lymphoblastoid cell lines (Bosch et al., 2007). The only known FAM90A 156 feature is a 19 amino acid motif that resembles a CCHC zinc-finger 157 domain that could be involved in DNA or RNA binding. According to 158 the human reference assembly, the family includes up to 25 members 159 per haploid genome, which share more than 93\% identity at the 160 nucleotide level (Fig. 1A). These 25 members are organized as tandem 161 array clusters along the REPD SDs, and as single copies on REPP SDs on 162 8p23.1, with the exception of FAM90A1, located on chromosome 163 12p13.31 (Fig. 1B). In the case of the HuRef assembly corresponding to 164 Craig Venter sequence, FAM90A gene copies map to chromosome 12165 (GenBank: CM00473), chromosome 8 (GenBank: CM000469) as well 166 as to chromosome 4 (GenBank: CM000465) (Levy et al., 2007). 167

The FAM90A gene family is only present in primates and it has 168 experienced an increase in copy number, along the hominoid lineage. 169 In our previous study, we observed variability in the number of 170 FAM90A members among the 20 human individuals that we tested 171 qualitatively, by pulsed field gel electrophoresis and Southern 172 blotting.

\subsection{Intra- and inter-population FAM90A copy number variability}

174

Technical challenges for accurately typing copy number in the 175 FAM90A gene family are multiple. Specific assays to determine the 176 presence or the absence for all the 25 FAM90A genes in a certain 177 individual are not feasible due to the high level of sequence identity 178 they share. Moreover, absolute quantification using large-scale 179 methods such as multiple ligation probe amplification (MLPA) is not 180 useful as it is not possible to discriminate the large number of gene 181 copies. Thus, we analyzed intra- and inter-population variability for 182 FAM90A CNV using a real-time $\mathrm{PCR}$ relative quantification method in 183 a sample of 260 HapMap individuals from three different ethnic 184 populations. 
B HsaCopy1 HsaCopy 2 HsaCopy 3 HsaCopy4 HsaCopy 5 HsaCopy6

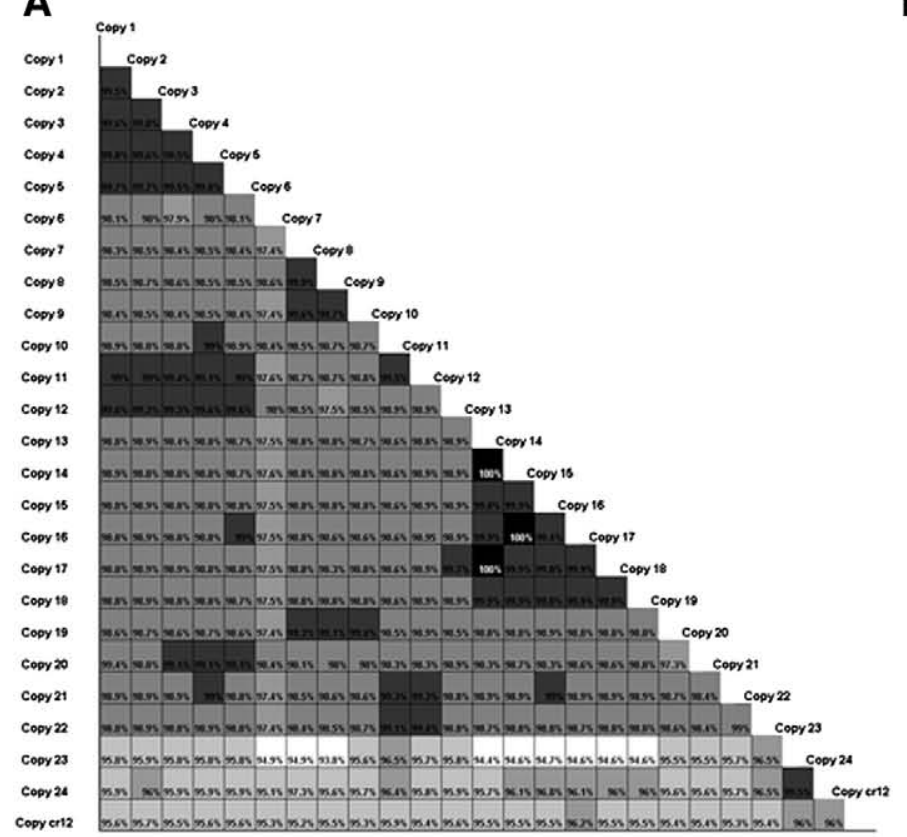

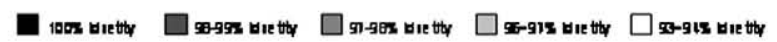

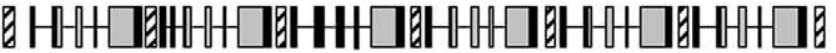

CLUSTER A
Chromosomespos.1

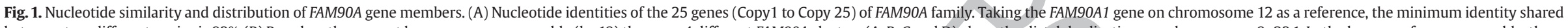

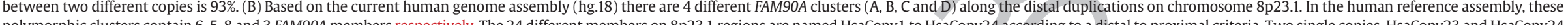

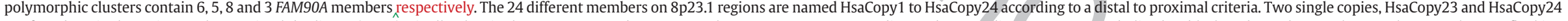

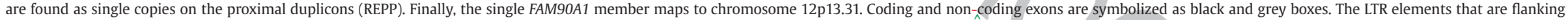
FAM90A members are depicted as striped boxes.

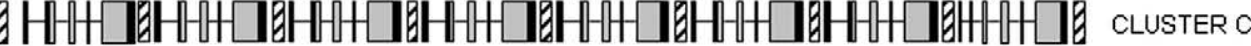

HsaCopy 20 HsaCopy 21 HsaCopy 22

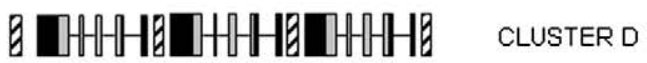

\begin{tabular}{|c|c|c|}
\hline 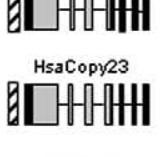 & $\begin{array}{c}\text { Chromosome 8p23.1 } \\
\text { REPP }\end{array}$ & $\begin{array}{l}\square \text { Non-coding exon } \\
\square \text { Coding exon } \\
\square \text { LTR }\end{array}$ \\
\hline
\end{tabular}

\section{FAM90A1}

LTR 
A

\section{FAMS0A Gene}

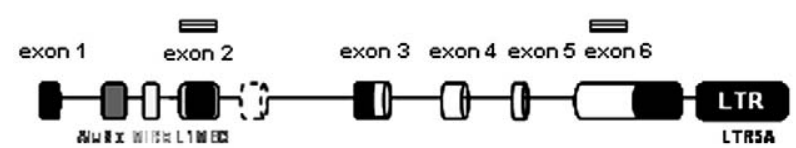

B

INTER-POPULATION COPYNUMBER VARIABLITY OF FAM90A

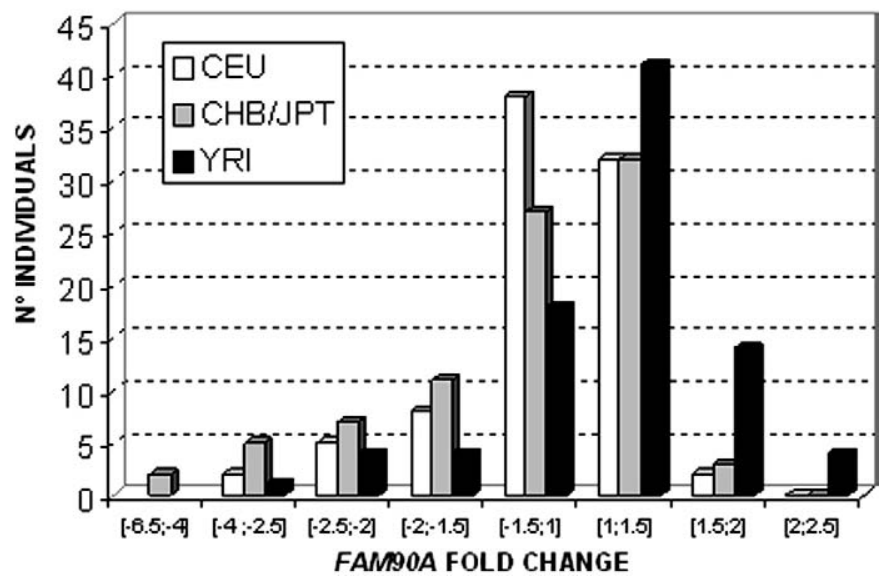

Fig. 2. Analysis of population variability of $\mathrm{CNV}$ at the FAM90A gene. (A) FAM90A gene structure. Filled boxes correspond to non-translated exons and open boxes to the coding sequence. The alternatively spliced exon is represented by a dotted line. Repetitive elements are symbolized as gray and black rectangles. Probes targeting exons 2 and 6 are represented as striped boxes. (B) Variability in FAM90A copy number among 260 HapMap individuals. $X$ axis represents 8 different intervals according to the fold-change values obtained using the mean of the $\Delta \Delta \mathrm{Cp}$ of UPL probe \#29 and UPL probe \#79. On the $Y$ axis, the number of individuals of the three populations included in each interval is represented.
Two different assays targeting the second and the last exon of FAM90A genes were used for quantification analysis (Fig. 2A; Supplementary Table 2). Calculations were based on the NA12892
CEU individual as the average sample regarding FAM90A copy number. 189 Inter-population differences are shown in Fig. 2B where the CHB/JPT 190 population shows the lower FAM90A copy number and the YRI 191 population shows the higher FAM90A copy number and with 192 statistically significant differences in FAM90A copy number with 193 respect to CEU and $\mathrm{CHB} / \mathrm{JPT}(p<0.0001)$.

Real-time PCR using the Universal Probe Library (Roche, Man- 195 nheim) revealed that the method is valid and accurate for quantifica- 196 tion since the two probes used, successfully replicated the values 197 regarding FAM90A relative copy number in all samples (Fig. 3A). 198 Moreover, only the YRI population showed statistically significant 199 intra-population differences regarding FAM90A; these results are 200 concordant with previous reports using HapMap samples, where the 201 YRI individuals were the most heterogeneous in comparison to other 202 populations (Foster and Sharp, 2004). We confirm that FAM90A 203 constitutes an example of a CNV with dramatic variability among 204 individuals, as the current genome assembly contains up to 25205 members of this gene family, and real-time quantitative PCR in 206 HapMap samples varied between a -6.3 and 2.1 fold increase in 207 FAM90A copy number, with respect to the reference sample. The 208 analysis of FAM90A as a CNV represents a good example of how 209 genomic regions flanked by SDs, such as the 8p23.1 region, can give 210 rise to highly polymorphic CNVs. It is widely accepted that such 211 genomic regions enhance variability of CNVs (Sebat et al., 2004; Sharp 212 et al., 2005), leading to different rearrangements by non-allelic homo- 213 logous recombination, thus increasing or decreasing the copy number 214 of the intervening sequence.

\subsection{Correlation between FAM90A copy number and expression levels 216}

To analyze the possible correlation between FAM90A copy number 217 and FAM90A expression levels, we utilized transcriptional data from 218 the genome-wide expression arrays analyzed by Stranger et al. (2007); 219 they produced a dataset of gene expression from EBV-transformed 220 lymphoblastoid cell lines from HapMap individuals. Positive correla- 221 tion was detected between FAM90A copy number and expression 222 levels $\left(r^{2}=0.05, p=0.002\right.$ ) (Fig. $\left.3 \mathrm{~B}\right)$; however, the correlation is weak 223 and population independent, indicating that other factors might be 224
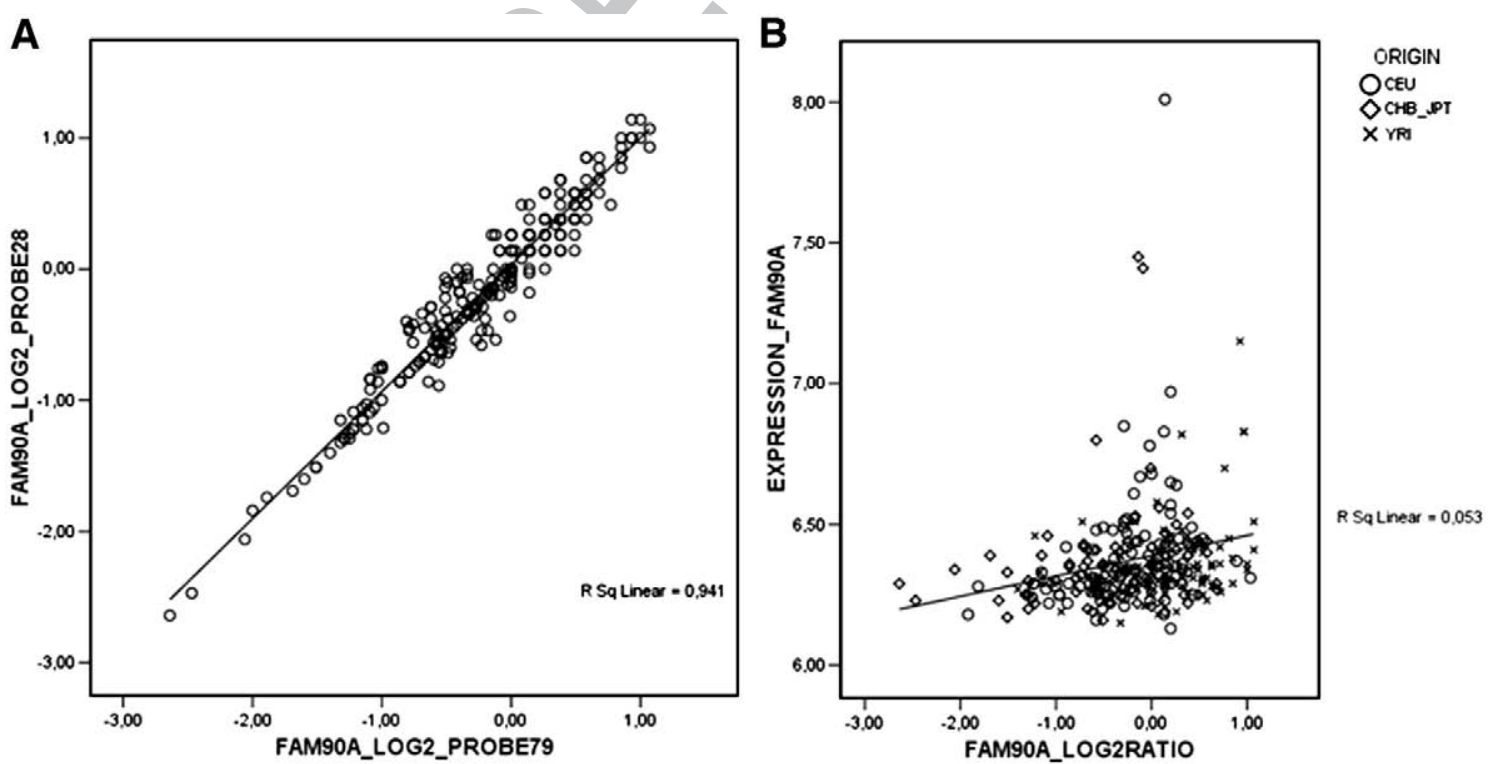

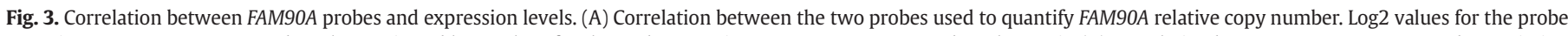

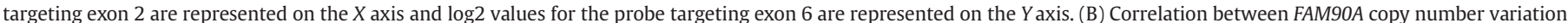

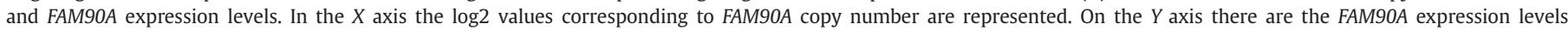

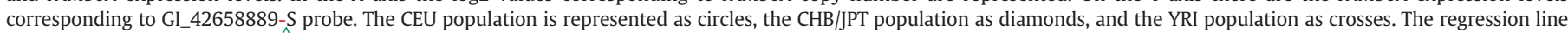
between FAM90A copy number and expression levels is depicted. 
controlling FAM90A expression levels. These results are in agreement with data for other CNVs in this chromosome region. It has also been shown that DEFA1/3 genes do not have correlation between copy number and expression levels (Aldred et al., 2005). Moreover, whole genome studies have revealed that between $8 \%$ and $18 \%$ of the heritable variation in transcript levels is due to gene copy number (Stranger et al., 2007), indicating that approximately $80 \%$ of this variation is copy number independent. However, this study was based on a limited number of CNVs and also the definition of their boundaries was not precise (Redon et al., 2006). Previous studies have shown that increased transcription levels associated to a reduced copy number are also possible (Stranger et al., 2007). Thus, the low correlation $\left(r^{2}=0.05, p=0.002\right)$ found between gene dosage and expression levels could be due to a complex regulation at the transcription level of FAM90A mRNAs. Indeed, heritable trans-acting factor variation has been found to be common and is expected to account for a high proportion of gene expression variability between individuals (Morley et al., 2004). Another feasible explanation for the low correlation found, could be that although the HapMap repository represents an enormous resource for genetic studies, lymphoblastoid cell lines might not be the main cell type where expression levels of FAM90A are being affected by gene dosage. Similar correlation studies in gene families, which have rapidly expanded in the hominoid lineage, employing appropriate tissues should help to further understand how gene copy number affects transcriptional levels, and therefore its evolutionary constraints.

\section{Acknowledgements}

Financial support was received from the Department of Universities, Research and Information Society (2005SGR00008) ("Generalitat de Catalunya"), from the European Union AnEUploidy project (grant number 037627). N. B. is a recipient of a BEFI fellowship from "Instituto de Salud Carlos III FIS-ISCIII"; J.M.M. was supported by the CRG under project SAF2002-00799 (Spanish Ministry of Science and Education) and by fellowship of the Danone Institute (Spain).

\section{Appendix A. Supplementary data}

Supplementary data associated with this article can be found, in the online version, at doi:10.1016/j.gene.2008.05.003.

\section{References}

Aitman, T.J., 2006. Copy number polymorphism in Fcgr3 predisposes to glomerulonephritis in rats and humans. Nature 439, 851-855.

Aldred, P.M., Hollox, E.J., Armour, J.A., 2005. Copy number polymorphism and expression level variation of the human \{alpha\}-defensin genes DEFA1 and DEFA3. Hum. Mol. Genet. 14, 2045-2052.

Ballana, E., Gonzalez, J.R., Bosch, N., Estivill, X., 2007. Inter-population variability of DEFA3 gene absence: correlation with haplotype structure and population variability. BMC Genomics 8, 14.
Beckmann, J.S., Estivill, X., Antonarakis, S.E., 2007. Copy number variants and genetic 271 traits: closer to the resolution of phenotypic to genotypic variability. Nat. Rev. 272 Genet. 8, 639-646.

Bosch, N., et al., 2007. Characterization and evolution of the novel gene family FAM90A 274 in primates originated by multiple duplication and rearrangement events. Hum. 275 Mol. Genet. 16, 2572-2582.

Estivill, X., Armengol, L., 2007. Copy number variants and common disorders: filling the 277 gaps and exploring complexity in genome-wide association studies. PLoS Genet. 3, 278 1787-1799.

Fanciulli, M., et al., 2007. FCGR3B copy number variation is associated with 280 susceptibility to systemic, but not organ-specific, autoimmunity. Nat. Genet. 39, 281 721-723.

Fellermann, K., et al., 2006. A chromosome 8 gene-cluster polymorphism with low 283 human beta-defensin 2 gene copy number predisposes to Crohn disease of the 284 colon. Am. J. Hum. Genet. 79, 439-448.

Foster, M.W., Sharp, R.R., 2004. Beyond race: towards a whole-genome perspective on 286 human populations and genetic variation. Nat. Rev. Genet. 5, 790-796. 287

Gonzalez, E., et al., 2005. The influence of CCL3L1 gene-containing segmental 288 duplications on HIV-1/AIDS susceptibility. Science 307, 1434-1440. 289

Hollox, E.J., Armour, J.A., Barber, J.C., 2003. Extensive normal copy number variation of a 290 beta-defensin antimicrobial-gene cluster. Am. J. Hum. Genet. 73, 591-600. 291

Hollox, E.J., et al., 2008. Psoriasis is associated with increased beta-defensin genomic 292 copy number. Nat. Genet. 40, 23-25. 293

Jakobsson, M., et al., 2008. Genotype, haplotype and copy-number variation in world- 294 wide human populations. Nature 451, 998-1003. 295

Levy, S., et al., 2007. The diploid genome sequence of an individual human. Plos Biol. 296 5 (10), e254.

Linzmeier, R.M., Ganz, T., 2005. Human defensin gene copy number polymorphisms: 298 comprehensive analysis of independent variation in alpha- and beta-defensin 299 regions at 8p22-p23. Genomics 86, 423-430.

Lynch, M., Conery, J.S., 2000. The evolutionary fate and consequences of duplicate genes. 301 Science 290, 1151-1155. 302

Morley, M., et al., 2004. Genetic analysis of genome-wide variation in human gene 303 expression. Nature 430, 743-747.

Nguyen, D.Q., Webber, C., Ponting, C.P., 2006. Bias of selection on human copy-number 305 variants. PLoS Genet. 2, e20. 306

Norton, H.L., et al., 2007. Genetic evidence for the convergent evolution of light skin in 307 Europeans and East Asians. Mol. Biol. Evol. 24, 710-722. 308

Perry, G.H., et al., 2007. Diet and the evolution of human amylase gene copy number 309 variation. Nat. Genet. 39, 1256-1260. 310

Perry, G.H., et al., 2008. The fine-scale and complex architecture of human copy-number 311 variation. Am. J. Hum. Genet. 82, 685-695. 312

Pfaffl, M.W., 2001. A new mathematical model for relative quantification in real-time 313 RT-PCR. Nucleic Acids Res. 29, e45. $\quad{ }^{\wedge} 314$

Redon, R., et al., 2006. Global variation in copy number in the human genome. Nature 315 444, 444-454. 316

Sebat, J., et al., 2004. Large-scale copy number polymorphism in the human genome. 317 Science 305, 525-528.

Sharp, A.J., et al., 2005. Segmental duplications and copy-number variation in the 319 human genome. Am. J. Hum. Genet. 77, 78-88. 320

Stranger, B.E., et al., 2007. Relative impact of nucleotide and copy number variation on 321 gene expression phenotypes. Science 315, 848-853.

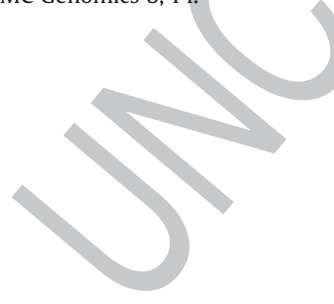

